\title{
Circular motion in NUT space-time
}

\author{
Paul I. Jefremov and Volker Perlick \\ ZARM, University of Bremen, 28359 Bremen, Germany. \\ Email: paul.jefremow@zarm.uni-bremen.de, volker.perlick@zarm.uni-bremen.de
}

August 2016

\begin{abstract}
We consider circular motion in the NUT (Newman-Unti-Tamburino) space-time. Among other things, we determine the location of circular time-like geodesic orbits, in particular of the innermost stable circular orbit (ISCO) and of the marginally bound circular orbit. Moreover, we discuss the von Zeipel cylinders with respect to the stationary observers and with respect to the Zero Angular Momentum Observers (ZAMOs). We also investigate the relation of von Zeipel cylinders to inertial forces, in particular in the ultra-relativistic limit. Finally, we generalise the construction of thick accretion tori ("Polish doughnuts") which are well known on the Schwarzschild or Kerr background to the case of the NUT metric. We argue that, in principle, a NUT source could be distinguished from a Schwarzschild or Kerr source by observing the features of circular matter flows in its neighbourhood.
\end{abstract}

\section{Introduction}

The Newman-Unti-Tamburino (NUT) metric is a solution to the vacuum Einstein equation that generalises the Schwarzschild metric. Whereas the Schwarzschild metric depends only on one parameter, $M$, which is to be interpreted as a mass, the NUT metric involves a second parameter, $n$, which is called the NUT charge or the gravitomagnetic charge. The solution was found by Newman, Tamburino and Unti [1] in 1963. Just as the the Schwarzschild solution, it may either be joined to an interior perfect fluid solution (see Bradley et al. [2]) or considered as a black-hole solution. In the latter case, the region beyond the horizon is isometric to Taub's cosmological vacuum solution [3]; therefore, the space-time that results from extending the NUT space-time over the horizon is known as the Taub-NUT solution.

Since the discovery of the NUT metric its physical relevance is a matter of debate. Here we have to face the problem that solving Einstein's (vacuum) field equation only gives us a local expression for the metric; there are different ways of how to construct a global space-time from this local form of the metric and these different ways may lead to quite different physical interpretations. Misner [4] has demonstrated how to construct a global NUT space-time that is regular everywhere outside the horizon and spherically symmetric. The price he had to pay was that the time coordinate had to be made periodic, so in Misner's version of the NUT space-time through each event 
outside the horizon there is a closed time-like curve. If one is not willing to accept such a violation of causality, one may resort to a global version of the NUT metric suggested by Bonnor [5]. In his interpretation there is a singularity on the half-axis $\theta=\pi$ which may be interpereted as a rotating massless rod. Closed time-like curves exist only in a neighbourhood of this singularity which may be arbitrarily small, so the situation is not worse than in the space-time of a rapidly rotating straight string which is widely accepted as physically relevant. As a generalisation of Bonnor's construction, Manko and Ruiz [6] have demonstrated that one may introduce an additional parameter $C$ into the NUT metric that allows to distribute the singularity over both half-axes $\theta=0$ and $\theta=\pi$ in a symmetric or asymmetric way. Also, we mention the possibility of superimposing two NUT charges of opposite sign to get a metric that is regular everywhere except between the two sources on the axis, see McGuire and Ruffini [7].

It is certainly fair to say that the properties of the NUT metric, in any interpretation, are somewhat unusual if not pathological. In an often quoted article, Misner [8] called it a "counter-example to almost anything". On the other hand, in the last hundred years we have learned that exact solutions to Einstein's (vacuum) field equation should be taken seriously. The Schwarzschild "singularity" at $r=2 M$ was considered as unphysical for many years; now we have strong evidence that black holes really exist and that the Schwarzschild metric (and more generally the Kerr metric) describes Nature correctly. Therefore, we believe that also the NUT metric should be considered as a hypothetical candidate for objects that exist in Nature. If one is willing to accept this idea as a working hypothesis, one has to investigate the question of how a NUT source could be detected, i.e., by what observational means we would know a NUT source if we see one. As for all astrophysical objects, in principle there are two such means: One could observe the influence on light (i.e., the lensing features) or the influence on massive particles. The lensing features of NUT objects have been comprehensively discussed by Nouri-Zonoz and Lynden-Bell [9]; an analytical formula for the shadow of a NUT black hole, which is a particular lensing feature, is contained as a special case in the work of Grenzebach et al. [10]. The motion of massive particles in the NUT space-time was studied in a mathematically very elaborate paper by Kagramanova et al. [11]; moreover, Hackmann and Lämmerzahl [12] calculated an upper bound for the NUT charge of the Sun by analysing the motion of Mercury. However, as to particle motion around compact NUT sources, in particular around NUT black holes, we believe that the question of how to relate the mathematical results to observational features has not yet been completely answered. It is the purpose of this paper to fill this gap. For the sake of simplicity, and because we believe that this case is particularly relevant, we restrict to circular particle motion.

The paper is organised as follows. In Section 2 we summarise some basic features of the NUT metric. In particular, we outline the differences between Misner's and Bonnor's interpretation and the relevance of the Manko-Ruiz parameter. In Section 3 we summarise some results on circular geodesics in the NUT space-time that will be used later. In Section 4 we discuss the so-called von Zeipel cylinders in the NUT space- 
time which, in our view, are very useful for illustrating circular motion. Section 5 gives a short summary of how the von Zeipel cylinders are related to inertial forces. Finally, in Section 6 we discuss a certain class of thick accretion tori, known as Polish doughnuts, around a NUT source. These exact analytical solutions of the Euler equation for a perfect fluid motion have been studied before on Schwarzschild and Kerr spacetimes, but not, to the best of our knowledge, on NUT space-time. We will see that the Polish doughnuts around a NUT source are qualitatively different from Polish doughnuts around a Schwarzschild or Kerr source, so the observation of such accretion tori allows, in principle, to discriminate between a NUT source and a Schwarzschild or Kerr source.

Our conventions are as follows. Throughout the paper we use units making $c$ and $G$ equal to unity. Greek indices are taking values 0,1,2,3 and Einstein's summation convention is used. Our choice of signature is $(+---)$.

\section{NUT space-time}

The Newman-Unti-Tamburino (NUT) metric is a solution to the vacuum Einstein equation that depends on two parameters, a mass $M$ and a gravitomagnetic charge (NUT parameter) n. It was discovered by Newman, Tamburino and Unti [1] in 1963, a detailed discussion can be found, e.g., in the book by Griffiths and Podolský [13]. In spherical coordinates $(t, r, \theta, \phi)$ the NUT metric reads

$$
g_{\mu \nu} d x^{\mu} d x^{\nu}=\frac{\Delta}{\Sigma}(\mathrm{d} t-2 n(\cos \theta+C) \mathrm{d} \phi)^{2}-\frac{\Sigma}{\Delta} \mathrm{d} r^{2}-\Sigma\left(\mathrm{d} \theta^{2}+\sin ^{2} \theta \mathrm{d} \phi^{2}\right)
$$

where

$$
\Delta=r^{2}-2 M r-n^{2}, \quad \Sigma=r^{2}+n^{2} .
$$

We have written the metric in the general form, also allowing for an additional parameter $C$ which was introduced by Manko and Ruiz [6]. This parameter may take any real value. The original version of the NUT metric is the metric (1) with $C=-1$. We will discuss the relevance of the parameter $C$ below.

Whereas the Schwarzschild space-time has a curvature singularity at $r=0$, in the NUT space-time with $n \neq 0$ the radius coordinate may range from $-\infty$ to $\infty$. (As an alternative, one may assume that the metric (1) is valid only for $r_{*}<r$, with some radius value $r_{*}$, and that at $r=r_{*}$ it is matched to an interior metric, e.g. to a perfect fluid solution of Einstein's field equation, see Bradley et al. [2]. However, we will not consider this case here.) The metric coefficient $g_{r r}$ becomes infinite at two radius values where $\Delta=0$. These are coordinate singularities which indicate horizons. We shall restrict our consideration to the domain of outer communication, i.e., to the region outside of the outer horizon,

$$
r_{\text {hor }}<r<\infty, \quad r_{\text {hor }}=M+\sqrt{M^{2}+n^{2}} .
$$

The adjacent region $M-\sqrt{M^{2}+n^{2}}<r<M+\sqrt{M^{2}+n^{2}}$, which will be of no interest to us, is known as the Taub region because there the space-time is isometric to Taub's solution [3] to Einstein's vacuum field equation. 
The NUT metric can be generalised to the Kerr-NUT metric which involves, in addition to $M$ and $n$, a specific angular momentum (Kerr parameter) $a$. The Kerr-NUT metric reads (cf. Griffiths and Podolský [13])

$$
g_{\mu \nu} d x^{\mu} d x^{\nu}=\frac{\Delta^{\prime}}{\Sigma^{\prime}}(\mathrm{d} t-\chi \mathrm{d} \phi)^{2}-\frac{\Sigma^{\prime}}{\Delta^{\prime}} \mathrm{d} r^{2}-\Sigma^{\prime} \mathrm{d} \theta^{2}-\frac{\sin ^{2} \theta}{\Sigma^{\prime}}\left(a \mathrm{~d} t-\left(\Sigma^{\prime}+a \chi\right) \mathrm{d} \phi\right)^{2}
$$

where $\Delta^{\prime}=r^{2}-2 M r+a^{2}-n^{2}, \Sigma^{\prime}=r^{2}+(n+a \cos \theta)^{2}$ and $\chi=a \sin ^{2} \theta-2 n(\cos \theta+C)$. This metric with $M>0$ describes a black hole provided that $M^{2}+n^{2} \geq a^{2}$. The outer horizon is at $r_{\text {hor }}=M+\sqrt{M^{2}+n^{2}-a^{2}}$. In this paper we will restrict to the NUT metric but we will occasionally refer, for the sake of comparison, to the Kerr metric, i.e., to the metric (4) with $n=0$. A common feature of the NUT and of the Kerr metric is that they are stationary but non-static, because of the non-vanishing $g_{t \phi}$ term. A major difference is in the fact that the Kerr metric is invariant under a transformation $\theta \mapsto \pi-\theta$ whereas the NUT metric is not. Thus, the NUT metric is not symmetrical with respect to an equatorial plane as will be clearly seen by several features to be discussed below.

We will now discuss the physical relevance of the Manko-Ruiz parameter $C$. Whereas the parameters $M$ and $n$ characterise invariant properties of the NUT metric, $C$ is to a certain extent subject to coordinate transformations. First we observe that a coordinate transformation

$$
\bar{t}=t, \quad \bar{\phi}=-\phi, \quad \bar{\theta}=\pi-\theta, \quad \bar{r}=r
$$

transforms $C$ to $\bar{C}=-C$. This demonstrates that NUT metrics with $C$ and $-C$ are globally isometric, where the isometry is given by a simultaneous inversion of the $\phi$ coordinate and reflection at the equatorial plane. Moreover, a coordinate transformation

$$
t^{\prime}=t-2 n\left(C-C^{\prime}\right) \phi, \quad \phi^{\prime}=\phi, \quad \theta^{\prime}=\theta, \quad r^{\prime}=r
$$

transforms a NUT metric with $C$ to a NUT metric with $C^{\prime}$, for any real numbers $C$ and $C^{\prime}$. However, (6) is not a globally well defined transformation unless we assume that $t$ is periodic with period $4\left|n\left(C-C^{\prime}\right)\right| \pi$. The reason is that $\phi$ is assumed to be periodic with period $2 \pi$. We may summarise these observations in the following way: If we are not willing to make the time coordinate periodic, NUT space-times with MankoRuiz parameters $C$ and $C^{\prime}$ are non-isometric and, thus, physically distinct if $|C| \neq\left|C^{\prime}\right|$. However, they are locally isometric, as demonstrated by (6), on any neighbourhood that does not contain a complete $\phi$ line. If the time coordinate has period $T$, NUT spacetimes with Manko-Ruiz parameters $C$ and $C^{\prime}$ are globally isometric if $4\left|n\left(C-C^{\prime}\right)\right| \pi=T$.

The Manko-Ruiz parameter is of particular relevance in view of the fact that the NUT metric features a singularity on the axis which is different from the trivial coordinate singularity of spherical polars that is familiar even from flat space. We can read this from the contravariant time-time component of the metric which can easily be calculated from (1),

$$
g^{t t}=\frac{4 n^{2}(\cos \theta+C)^{2}}{\Sigma \sin ^{2} \theta}+\frac{\Sigma}{\Delta} .
$$


While the second term is finite everywhere on the domain of outer communication, the first one diverges for $\theta \rightarrow 0$ unless $C=-1$ and for $\theta \rightarrow \pi$ unless $C=1$. There are two different ways of how to interpret this singularity, depending on whether or not one is willing to make the time coordinate periodic.

The first interpretation was brought forward by Misner [4]. He considered the metric (1) with $C=-1$ which is regular except on the negative half-axis, $\theta=\pi$. By a coordinate transformation (6) with $C^{\prime}-C=2$ he got a NUT metric with $C^{\prime}=1$ which is regular except on the positive half-axis, $\theta=0$. This transformation requires the time coordinate to be periodic with period $8|n| \pi$. Cutting out the singular half-axis from each of the two copies gives two coordinate patches that can be glued together to give a NUT space-time that is regular on both half-axes. This space-time is globally spherically symmetric, i.e., there is no distinguished axis. Of course, assuming that the time coordinate is periodic means that there is a closed time-like curve through

any event of the domain of outer communication, i.e., that the space-time violates the causality condition in an extreme way. However, one could argue that this is of no practical relevance if one assumes the period $T=8|n| \pi$ to be very large. Misner's construction can be generalised to gluing together a NUT metric with $C$ and a NUT metric with $C^{\prime}=-C$, for any $C \neq 0$, provided that the time coordinate is periodic with period $8|n C| \pi$. However, in the case $|C| \neq 1$ this is not very interesting because the resulting space-time still has a singularity on the axis.

The second interpretation, which goes back to Bonnor [5], is based on the assumption that the $t$ coordinate is not periodic. Then (6) is not a globally allowed transformation and, for any choice of $C$, we have a true singularity on at least one halfaxis. Bonnor interpreted this singularity as a massless spinning rod. He considered only the case that $C=-1$ where the singularity is on the negative half-axis. For $C=1$ it is on the positive half-axis, for $C=0$ it is distributed symmetrically and for any other value of $C$ asymmetrically on both half-axes. In Bonnor's version the NUT metric is locally spherically symmetric, near any point off the axis, but not globally in contrast to Misner's version with $|C|=1$. As we read from (1) that the $\phi$ lines are spacelike near the singularity, also in Bonnor's interpretation the NUT metric contains closed time-like curves in the domain of outer communication. However, by choosing $|n|$ and $|C|$ sufficiently small one may restrict them to a narrow neighbourhood of the axis so that they may be considered as irrelevant for possible motions of observers.

\section{Time-like circular geodesics}

It is well known that in the NUT space-time the Hamilton-Jacobi equation for the geodesics is separable which results in the following first-order equations for the timelike geodesics, cf. Kagramanova et al. [11]:

$$
\dot{r}^{2}=R:=\frac{1}{4 M^{2}}\left(\Sigma^{2} E^{2}-\Delta\left(r^{2}+L^{2}+K\right)\right),
$$




$$
\begin{aligned}
& \dot{\theta}^{2}=\Theta:=\frac{1}{4 M^{2}}\left(K+L^{2}-n^{2}-\frac{(L-2 n E(\cos \theta+C))^{2}}{\sin ^{2} \theta}\right), \\
& \dot{\phi}=\frac{1}{2 M}\left(\frac{L-2 n E(\cos \theta+C)}{\sin ^{2} \theta}\right), \\
& \dot{t}=\frac{1}{2 M}\left(E \frac{\Sigma^{2}}{\Delta}+2 n(\cos \theta+C) \frac{(L-2 n E(\cos \theta+C))}{\sin ^{2} \theta}\right) .
\end{aligned}
$$

Here $E$ is the energy, $L$ is the $z$ component of the angular momentum and $K$ is the Carter constant. (Note that Kagramanova et al. [11] use a Carter constant $k$ which is related to our $K$ by $4 M^{2} k=K$.) A dot denotes derivative with respect to the Mino time which is not an affine parameter. By a coordinate transformation (6) with $C^{\prime}=0$, which in any case is well-defined locally near any point off the axis, one can get rid of the Manko-Ruiz parameter $C$ in (1). This transforms the equations for time-like geodesics to

$$
\begin{aligned}
& \dot{r}^{2}=\frac{1}{4 M^{2}}\left(\Sigma^{2} E^{\prime 2}-\Delta\left(r^{2}+L^{\prime 2}+K^{\prime}\right)\right), \\
& \dot{\theta}^{2}=\frac{1}{4 M^{2}}\left(K^{\prime}+L^{\prime 2}-n^{2}-\frac{\left(L^{\prime}-2 n E^{\prime} \cos \theta\right)^{2}}{\sin ^{2} \theta}\right), \\
& \dot{\phi}=\frac{1}{2 M}\left(\frac{L^{\prime}-2 n E^{\prime} \cos \theta}{\sin ^{2} \theta}\right), \\
& \dot{t}^{\prime}=\frac{1}{2 M}\left(E^{\prime} \frac{\Sigma^{2}}{\Delta}+2 n \cos \theta \frac{\left(L^{\prime}-2 n E^{\prime} \cos \theta\right)}{\sin ^{2} \theta}\right)
\end{aligned}
$$

where the new constants of motion are related to the old ones from (8), (9), (10) and (11) by

$$
E^{\prime}=E, \quad L^{\prime}=L-2 n C E, \quad K^{\prime}=K+4 n C E L-4 n^{2} C^{2} E^{2} .
$$

We see that the values of the constants of motion $L$ and $K$ assigned to a particular geodesic depend on $C$. However, as curves in the space-time the time-like geodesics are locally independent of $C$ in the sense that, near any point off the axis, they can be diffeomorphically mapped from a space-time with arbitrary $C$ onto a space-time with $C^{\prime}=0$. As this diffeomorphism involves only a transformation of the $t$ coordinate, the spatial projections $(\phi(s), \theta(s), r(s))$ of time-like geodesics are globally independent of $C$.

It is now our goal to determine all circular time-like geodesics in this space-time. In particular we will determine the location of the innermost stable circular orbit and of the marginally bound orbit. These results will be of importance for us later and we believe that they have not been given correctly before.

As we said, we restrict to the domain of outer communication. It is our goal to find all circular time-like geodesics about the $z$-axis. All other circular time-like geodesics then follow immediately by applying all possible rotations. In space-times with a singularity on the axis (i.e., in all cases except in Misner's spherically symmetric 
NUT space-time with $|C|=1$ ), we have to omit all curves that pass through this singularity. Note that the spherical symmetry is broken only by the singularity on the axis: If this axis is removed, the hypersurfaces $r=$ constant are homogeneous under the flow of a three-dimensional Lie algebra of Killing vector fields that generate the rotation group, see the discussion in Bonnor's paper[5] for the case $C=-1$. This flow maps circular time-like geodesics onto circular time-like geodesics.

Circular geodesics about the $z$ axis have to satisfy the four equations $\dot{r}=0, \ddot{r}=0$, $\dot{\theta}=0$ and $\ddot{\theta}=0$. With the notation introduced in (8) and (9) these can be rewritten as

$$
R=0, \quad \frac{d R}{d r}=0, \quad \Theta=0, \quad \frac{d \Theta}{d \theta}=0 .
$$

These four equations allow us to express the constants of motion in terms of $r$ and $\theta$,

$$
\begin{aligned}
& E=\frac{\sqrt{\Sigma \Delta}}{\sqrt{\Sigma^{2}-4 n^{2} \Delta \tan \theta}}, \\
& L=\frac{\sqrt{4 \Sigma \Delta} n(1+C \cos \theta)}{\cos \theta \sqrt{\Sigma^{2}-4 n^{2} \Delta \tan \theta}}, \\
& K=n^{2}-\frac{4 \Sigma \Delta n\left((1+C \cos \theta)^{2}-\sin ^{2} \theta\right)}{\cos ^{2} \theta\left(\Sigma^{2}-4 n^{2} \Delta \tan \theta\right)} .
\end{aligned}
$$

Here we restrict to the case that $E>0$, i.e., that the tangent vector to the time-like geodesic points into the same half of the light-cone as $\partial_{t}$. (Note that, in contrast to the Kerr space-time, in the NUT space-time there is no ergoregion, i.e., $\partial_{t}$ is time-like on the entire domain of outer communication.) With $E, L$ and $K$ determined this way, (17) leaves us with one equation that gives us a relation between $r$ and $\theta$,

$$
\cos ^{2} \theta=\frac{4 n^{2}}{Q(r)}
$$

where

$$
Q(r):=\frac{6 n^{6} r+16 M^{2} n^{2} r^{3}-4 n^{4} r^{3}+6 n^{2} r^{5}+M\left(r^{6}+15 n^{4} r^{2}-15 n^{2} r^{4}-n^{6}\right)}{r \Delta^{2}},
$$

i.e., it gives us the location of the circular time-like geodesics. (21) reflects the fact that the NUT parameter breaks the symmetry with respect to the equatorial plane: The circular time-like orbits are not in the plane $\theta=\pi / 2$ if $n \neq 0$. (This can also be read from (18), (19) and (20).) They are rather located on two curved surfaces, given by the solution of (21) with $\cos \theta>0$ and $\cos \theta<0$, respectively. On one surface the motion is in the positive $\phi$ direction, on the other in the negative $\phi$ direction. $\ddagger$

We have already observed that the projections of time-like geodesics to threedimensional space are globally independent of the Manko-Ruiz parameter $C$. This is reflected by the fact that $C$ does not enter into equation (21).

$\ddagger$ Our result on the surfaces of circular motion is in disagreement with that of Chakraborty [14], who started his consideration of circular motion from the false assumption that the circular orbits lie in the plane $\theta=\frac{\pi}{2}$. 
Circular time-like geodesics exist for radii from infinity down to the radius of the photon sphere where the circular geodesic orbits become light-like. In the NUT spacetime, the radius of the photon sphere is given by

$$
4 r \Delta=\Sigma \frac{d \Delta}{d r}
$$

cf. Grenzebach et al. [10]. The unique solution outside the horizon is

$$
r_{\mathrm{ph}}=M+2\left(M^{2}+n^{2}\right)^{1 / 3} \operatorname{Re}\left((M+i n)^{1 / 3}\right)
$$

which reduces to the well known result $r_{\mathrm{ph}}=3 M$ in the Schwarzschild limit $n \rightarrow 0$. Note that in the NUT case the circular photon orbits are not great circles, i.e., that for $r \rightarrow r_{\mathrm{ph}}$ the angle $\theta$ as given by (21) does not go to $\pi / 2$.

However, not all the circular time-like geodesics between infinity and the photon sphere are stable. In order to find the radius of the innermost stable circular orbit (ISCO) we have to consider again the equations (18), (19), (20) and (21) for circular orbits, and we have to insert these expressions into the condition

$$
\frac{d^{2} R}{d r^{2}}=0
$$

which expresses marginal stability with respect to radial perturbations. This leads to the equation

$$
M r^{6}-6 M^{2} r^{5}-15 M n^{2} r^{4}+\left(4 M^{2} n^{2}-16 n^{4}\right) r^{3}+15 M n^{4} r^{2}-6 M^{2} n^{4} r-M n^{6}=0
$$

for the radius of the ISCO. This equation cannot be solved analytically but it can be verified that there is a unique real solution $r=r_{\mathrm{ms}}>r_{\text {hor }}$. In the limit $n \rightarrow 0$ it gives the well-known ISCO radius in the Schwarzschild space-time, $r_{\mathrm{ms}}=6 \mathrm{M}$. $\S$

Another important radius value is the radius of the marginally bound orbit which gives the lower bound for those orbits which do not go to infinity under small radial perturbations. From (18) we read that $E \rightarrow 1$ if $r \rightarrow \infty$. Therefore, the marginally bound orbit is found by solving the system of equations

$$
\left.R\right|_{E=1}=0,\left.\quad \frac{d R}{d r}\right|_{E=1}=0
$$

and is given by the expression

$$
\begin{aligned}
r_{\mathrm{mb}} & =M+\sqrt{M^{2}+n^{2}+\left(\frac{n^{2}\left(M^{2}+n^{2}\right)}{2 M}\right)^{2 / 3}} \\
& +\sqrt{2 M^{2}+2 n^{2}-\left(\frac{n^{2}\left(M^{2}+n^{2}\right)}{2 M}\right)^{2 / 3}+\frac{2 M^{4}+3 M^{2} n^{2}+n^{4}}{M \sqrt{M^{2}+n^{2}+\left(\frac{n^{2}\left(M^{2}+n^{2}\right)}{2 M}\right)^{2 / 3}}}}
\end{aligned}
$$

For $n \rightarrow 0$ this reduces to the well-known result $r_{\mathrm{mb}}=4 M$ for the marginally bound orbit in the Schwarzschild space-time.

$\S$ The equation (26) was obtained by Chakraborty [14] as a limiting case (with $a \rightarrow 0$ ) of his equation for marginally stable orbits in the Kerr-NUT space-time. However, he incorrectly assumed throughout his work that the circular orbits, and hence the ISCO, are in the equatorial plane $\theta=\pi / 2$. Actually, the $\theta$ coordinate of the ISCO is to be determined by inserting the ISCO radius into (21). 


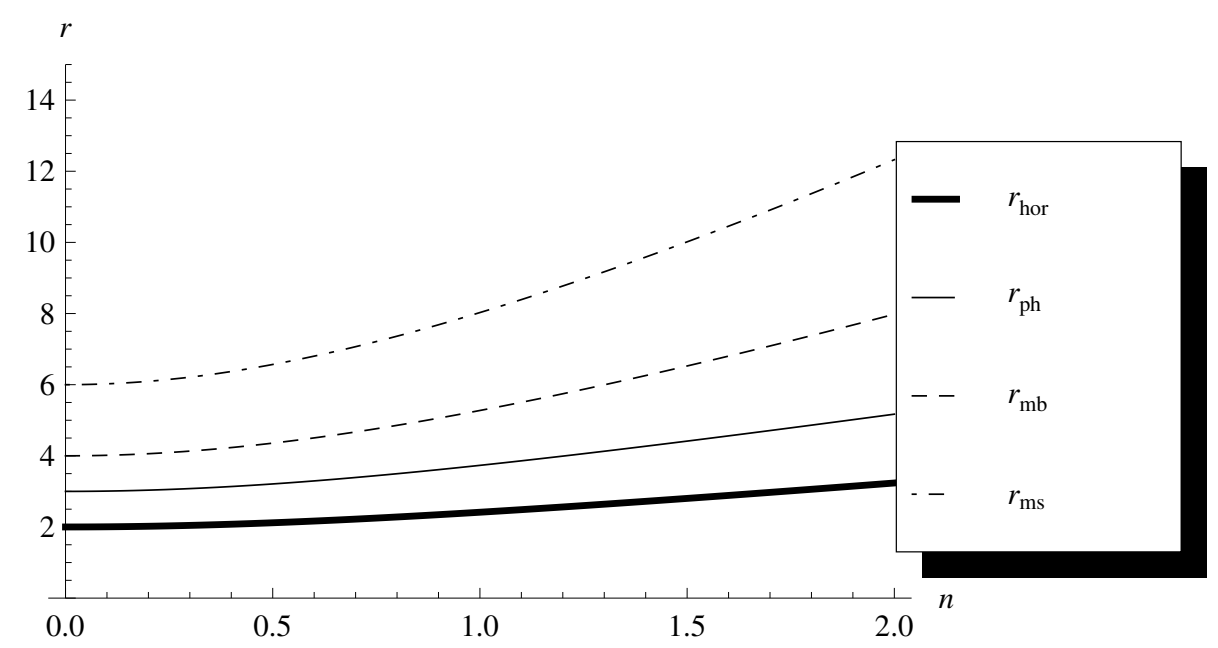

Figure 1. Dependence on the NUT parameter $n$ of the radii of the horizon $r_{\text {hor }}$, the photon sphere $r_{\mathrm{ph}}$, the marginally bound circular orbit $r_{\mathrm{mb}}$ and the marginally stable circular orbit (ISCO) $r_{\mathrm{ms}}$. The values of $n$ and $r$ are given in units of $M$.

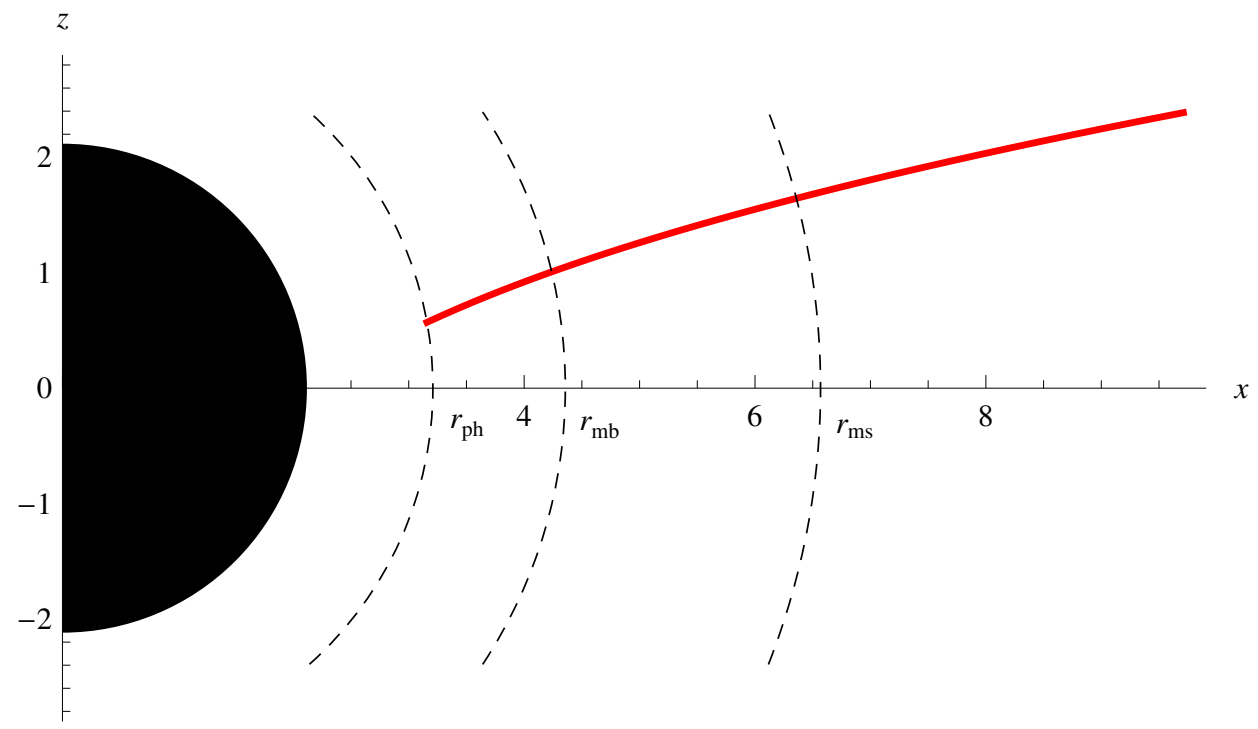

Figure 2. Location of time-like circular geodesics in the vicinity of a NUT black hole with $n=0.5 M$. On both axes $M$ is chosen as the unit. One of the two surfaces where geodesic circular motion about the $z$ axis is possible is marked by a thick (red) line. The other one is the mirror image with respect to the equatorial plane. Time-like circular geodesics exist only up to the photon sphere $r_{\mathrm{ph}}$; in the interval $r_{\mathrm{ph}}<r<r_{\mathrm{ms}}$ circular motion is unstable and for $r>r_{\mathrm{ms}}$ it is stable. The circular orbits with $r<r_{\mathrm{mb}}$ become unbound under radial perturbations whereas for $r>r_{\mathrm{mb}}$ they remain bound, see the text for further explanations.

The results on the photon sphere, the ISCO and the marginally bound orbit in the NUT space-time are summarised in Fig. 1. The location of the circular time-like geodesics is illustrated, for the case $n=0.5 M$, in Fig. 2 . 


\section{Von Zeipel cylinders}

In this section we want to study circular (in general non-geodesic) particle motion in the NUT space-time and we want to use the so-called von Zeipel cylinders for the sake of illustration. The relativistic notion of von Zeipel cylinders was introduced by Abramowicz [15] who showed that with the help of these cylinders a relativistic analogue to the classical (i.e., non-relativistic) von Zeipel theorem can be formulated.

Before specifying to the NUT space-time, we re-establish some basic facts about the von Zeipel cylinders that are valid in any axisymmetric and stationary space-time, so for the time being we just assume that we have a metric of the form

$$
g_{\mu \nu} d x^{\mu} d x^{\nu}=g_{t t} d t^{2}+2 g_{\phi t} d t d \phi+g_{\phi \phi} d \phi^{2}+g_{r r} d r^{2}+g_{\theta \theta} d \theta^{2}
$$

with two Killing vector fields $\partial_{t}$ and $\partial_{\phi}$. Note that there is a certain ambiguity in the choice of $\partial_{t}$ and $\partial_{\phi}$ because any linear combination with constant coefficients of two Killing vector fields is again a Killing vector field. We restrict this ambiguity by requiring that $\partial_{t}$ is time-like (on the entire domain of outer communication in the NUT spacetime and outside of the ergoregion in the Kerr space-time) and that the $\phi$ coordinate is periodic with period $2 \pi$. If the time coordinate is not periodic, this fixes $\partial_{t}$ up to an irrelevant constant factor and $\partial_{\phi}$ up to sign. If the time coordinate is periodic with period $4\left|n\left(C-C^{\prime}\right)\right| \pi$, we are still free to make coordinate transformations (6) which leave the vector field $\partial_{t}$ invariant but not the hypersurfaces $t=$ constant.

In a space-time of the form (29) where $\partial_{t}$ is time-like and $\phi$ is periodic with period $2 \pi$, circular motion about the $z$ axis is described by a four-velocity of the form

$$
u^{\mu} \partial_{\mu}=\frac{\partial_{t}+\Omega \partial_{\phi}}{\sqrt{g_{t t}+2 \Omega g_{t \phi}+\Omega^{2} g_{\phi \phi}}}
$$

where $\Omega$ is the angular velocity with respect to the stationary observers, i.e., observers moving on $t$ lines. $\Omega$ may be chosen as an arbitrary function of $r$ and $\theta$, subject only to the condition that $\partial_{t}+\Omega \partial_{\phi}$ be timelilke,

$$
g_{t t}+2 \Omega g_{t \phi}+\Omega^{2} g_{\phi \phi}>0 .
$$

We may interpret $u^{\mu} \partial_{\mu}$ as the four-velocity of a fluid, but in this section we will restrict to purely kinematical considerations and not assume any equation of motion.

If we define the energy and the $z$ component of the angular momentum in the usual way from the Lagrangian for free motion as $E=u_{t}$ and $L=-u_{\phi}$, the specific angular momentum is given as

$$
\ell=\frac{L}{E}=\frac{-\Omega g_{\phi \phi}-g_{t \phi}}{g_{t t}+\Omega g_{t \phi}} .
$$

The motion can be characterised either by giving $\Omega$ as a function of $r$ and $\theta$ or, equivalently, by giving $\ell$ as a function of $r$ and $\theta$, as (32) can be solved for $\Omega$,

$$
\Omega=\frac{\ell g_{t t}+g_{t \phi}}{-g_{\phi \phi}-\ell g_{t \phi}}
$$


If rewritten in terms of $\ell$, the condition (31) for time-like motion reads

$$
g_{\phi \phi}+2 g_{t \phi} \ell+g_{t t} \ell^{2}<0 .
$$

The von Zeipel radius (or radius of gyration) $\mathcal{R}$ was defined by Abramowicz and collaborators for the case of an axisymmetric and static metric (see in particular Abramowicz et al. [16]) by the definition

$$
\mathcal{R}^{2}=\frac{\ell}{\Omega}
$$

The surfaces $\mathcal{R}=$ constant are known as the von Zeipel cylinders. In the static case, $g_{t \phi}=0$, inserting (32) and (33) into (35) yields

$$
\mathcal{R}^{2}=\frac{-g_{\phi \phi}}{g_{t t}}
$$

On the domain where $\partial_{t}$ is time-like, the condition $g_{t \phi}=0$ implies that the right-hand side of (36) is non-negative and $\mathcal{R}$ is well-defined as a non-negative universal function of $r$ and $\theta$, independent of $\Omega$. On the Schwarzschild space-time this is the case on the entire domain of outer communication.

Definition (35) makes sense also in the more general case of an axisymmetric and stationary metric with $g_{t \phi} \neq 0$, such as the Kerr metric or the NUT metric. Then the von Zeipel radius $\mathcal{R}$ is given by the expression.

$$
\mathcal{R}^{2}=\frac{\ell}{\Omega}=\frac{-\Omega^{-1} g_{t \phi}-g_{\phi \phi}}{g_{t t}+\Omega g_{t \phi}}=\frac{-\ell g_{t \phi}-g_{\phi \phi}}{\ell^{-1} g_{t \phi}+g_{t t}} .
$$

The major difference in comparison to the case $g_{t \phi}=0$ is in the fact that now the function $\mathcal{R}$ depends on the motion. If the motion has been specified, by giving either $\Omega$ or $\ell$ as a function of $r$ and $\theta$, the von Zeipel radius is a well-defined function of $r$ and $\theta$ on the domain where the right-hand side of (37) is non-negative. This domain depends on the motion. Note that in flat space-time $\left(g_{t t}=1, g_{t \phi}=0, g_{\phi \phi}=-r^{2} \sin ^{2} \theta\right)$ we have $\mathcal{R}=r \sin \theta$, i.e., $\mathcal{R}$ is just the distance from the $z$ axis and the von Zeipel cylinders are indeed Euclidean cylinders in the three-dimensional space. As a consequence, if our axisymmetric and stationary space-time is asymptotically flat, the surfaces $\mathcal{R}=$ constant have the topology of cylinders if we are sufficiently far away from the axis. In the inner region of the space-time, however, their topology might be different.

For the NUT space-time, the relation (32) between $\ell$ and $\Omega$ specifies to

$$
\ell=\frac{\Omega\left(4 n^{2}(\cos \theta+C)-\frac{\Sigma^{2}}{\Delta} \sin ^{4} \theta\right)}{1-2 \Omega n(\cos \theta+C)}
$$

and the von Zeipel radius (37) reads

$$
\mathcal{R}^{2}=\frac{2 \ell n(\cos \theta+C)-4 n^{2}(\cos \theta+C)^{2}+\frac{\Sigma^{2}}{\Delta} \sin ^{4} \theta}{1-2 \ell^{-1} n(\cos \theta+C)} .
$$

With the help of these equations one easily verifies that the von Zeipel cylinders $\mathcal{R}=$ constant are independent of the Manko-Ruiz parameter $C$ in the following sense: On a NUT space-time with $C \neq 0$, the von Zeipel cylinders for a motion with angular 
velocity $\Omega$ and specific angular momentum $\ell$ coincide with the von Zeipel cylinders on a NUT space-time with the same $M$ and $n$ and $C=0$ for a motion with

$$
\Omega^{\prime}=\frac{\Omega}{1-2 n C \Omega}, \quad \ell^{\prime}=\ell-2 n C .
$$

We emphasise that the von Zeipel radius is a purely kinematic notion in the sense that it is well defined for any circular motion without reference to an equation of motion. However, it has particularly nice properties if we do assume a specific equation of motion. For the case that the Euler equation of a perfect fluid with a barotropic equation of state is satisfied, Abramowicz [15] has shown that the differential $d \Omega$ is a multiple of the differential $d \ell$ at all points where $d \ell \neq 0$. In other words, in this case $\Omega$ and thus $\mathcal{R}$ is constant on each non-degenerate surface $\ell=$ constant. This result is known as the relativistic von Zeipel theorem, cf. Rezzolla and Zanotti [17] for a detailed discussion.

Whereas (35) is the unanimously accepted definition of the von Zeipel radius in axisymmetric and static space-times, the generalisation to the axisymmetric and stationary case is not unambiguous. Abramowicz et al. [18] argued that in the latter case (with $g_{t \phi} \neq 0$ ) a modified von Zeipel radius $\tilde{\mathcal{R}}$ should be introduced by the equation

$$
\tilde{\mathcal{R}}^{2}=\frac{\tilde{\ell}}{\tilde{\Omega}}=\frac{g_{\phi \phi}^{2}}{g_{t \phi}^{2}-g_{t t} g_{\phi \phi}},
$$

where $\tilde{\Omega}$ denotes the angular velocity with respect to the zero angular momentum observers (ZAMOs),

$$
\tilde{\Omega}=\Omega-\omega, \quad \omega=-\frac{g_{t \phi}}{g_{\phi \phi}},
$$

and $\tilde{\ell}$ is the specific angular momentum with respect to the ZAMOs,

$$
\tilde{\ell}=\frac{\tilde{L}}{\tilde{E}}=\frac{L}{E-\omega L}=\frac{\ell}{1-\omega \ell} .
$$

The ZAMOs are observers whose worldlines are orthogonal to the hypersurfaces $t=$ constant. The name refers to the fact that these observers have $L=0$. In comparison to (37) the modified definition (41) has the obvious advantage that $\tilde{\mathcal{R}}$ is a universal function of $r$ and $\theta$, independent of the specific motion. Note that in the Kerr-NUT space-time $g_{t \phi}^{2}-g_{t t} g_{\phi \phi}$ is positive on the domain of outer communication, so the righthand side of (41) is non-negative there. However, when taking the square-root one has to be careful with the sign of $g_{\phi \phi}$. In the Kerr space-time, $g_{\phi \phi}$ is negative on the entire domain of outer communication, but in the NUT space-time there is a region near the singularity on the axis, depending on $C$, where $\partial_{\phi}$ is time-like. As on this region the hypersurfaces $t=$ constant are time-like, i.e., the ZAMOs would have to move at superluminal speed, it seems reasonable to omit this region. So we say that $\tilde{R}$ is defined only on that part of the space-time where the hypersurfaces $t=$ constant are spacelike and there we have

$$
\tilde{\mathcal{R}}=\frac{-g_{\phi \phi}}{\sqrt{g_{t \phi}^{2}-g_{t t} g_{\phi \phi}}} .
$$


Abramowicz et al. [18] argue that one should always work with $\tilde{\mathcal{R}}$ (which coincides, of course, with $\mathcal{R}$ in the case $g_{t \phi}=0$ ). Clearly, the fact that $\tilde{\mathcal{R}}$ is independent of the motion strongly supports this opinion. On the other hand, $\mathcal{R}$ is of some relevance also in the case $g_{t \phi} \neq 0$ because it is associated with the above-mentioned relativistic von Zeipel theorem. Moreover, the following observation, which follows immediately from the definitions, is of some importance: For a motion with $\ell=$ constant, the differential $d \mathcal{R}$ is a multiple of $d \Omega$, so in this case the surfaces $\mathcal{R}=$ constant are surfaces of constant $\Omega$. An analogous statement is true if we replace $\ell, \Omega$ and $\mathcal{R}$ with $\tilde{\ell}, \tilde{\Omega}$ and $\tilde{\mathcal{R}}$, respectively. This indicates that both $\mathcal{R}$ and $\tilde{\mathcal{R}}$ are useful quantities. $\|$ Henceforth we refer to the surfaces $\mathcal{R}=$ constant as to the "von Zeipel cylinders with respect to the stationary observers" and to the surfaces $\tilde{\mathcal{R}}=$ constant as to the "von Zeipel cylinders with respect to the ZAMOs".

We now specify the function $\tilde{\mathcal{R}}$, which is independent of the specific motion, for the NUT metric. Inserting the metric coefficients into (41) yields

$$
\tilde{\mathcal{R}}^{2}=\frac{\left(\Sigma^{2} \sin ^{2} \theta-4 n^{2} \Delta(\cos \theta+C)^{2}\right)^{2}}{\Delta \Sigma^{2} \sin ^{2} \theta} .
$$

We see that $\tilde{\mathcal{R}}$ depends on the Manko-Ruiz parameter $C$. The case $C=0$ plays a special role because in this case $\tilde{\mathcal{R}}^{2}$ is invariant under a transformation $\theta \mapsto \pi-\theta$, i.e., the von Zeipel cylinders with respect to the ZAMOs are symmetric with respect to the equatorial plane. For $n \rightarrow 0$ we get from (45) the well-known expression for the von Zeipel radius of the Schwarzschild metric,

$$
\tilde{\mathcal{R}}^{2}=\mathcal{R}^{2}=\frac{r^{4} \sin ^{2} \theta}{r^{2}-2 M r} .
$$

Fig. 3 illustrates the surfaces $\tilde{\mathcal{R}}=$ constant around a NUT black hole with $C=0$ and, for the sake of comparison, around a Schwarzschild black hole. We see that they are qualitatively quite similar. In particular, in both cases $\tilde{\mathcal{R}}$ is symmetric with respect to the equatorial plane. Note, however, that in the NUT case there is a region near the singularity on the axis where $g_{\phi \phi}>0$; as already indicated above, we say that $\tilde{\mathcal{R}}$ is not defined on this region. The situation is different for the NUT metric with $C \neq 0$, see Fig. 4. Then the symmetry with respect to the equatorial plane is broken. The case $|C|=1$ is special because then only one half-axis is singular; correspondingly, a region where $g_{\phi \phi}>0$ exists only near one half-axis. Here we consider Bonnor's interpretation of the NUT metric. With Misner's interpretation, the situation is more subtle. If the time coordinate is periodic, we may transform the hypersurfaces $t=$ constant, and thereby the ZAMOs, according to (6). In Misner's NUT space-time with $C= \pm 1$, which is regular on both half-axes, we have two families of ZAMOs and, correspondingly, two different functions $\tilde{\mathcal{R}}$. For either family of ZAMOs the domain of definition excludes a region near one of the two half-axes. As in this case the space-time is spherically symmetric, we may choose any axis for this construction, so there are infinitely many families of ZAMOs.

\| There are also other ways to define von Zeipel cylinders, see e. g. [19]. 

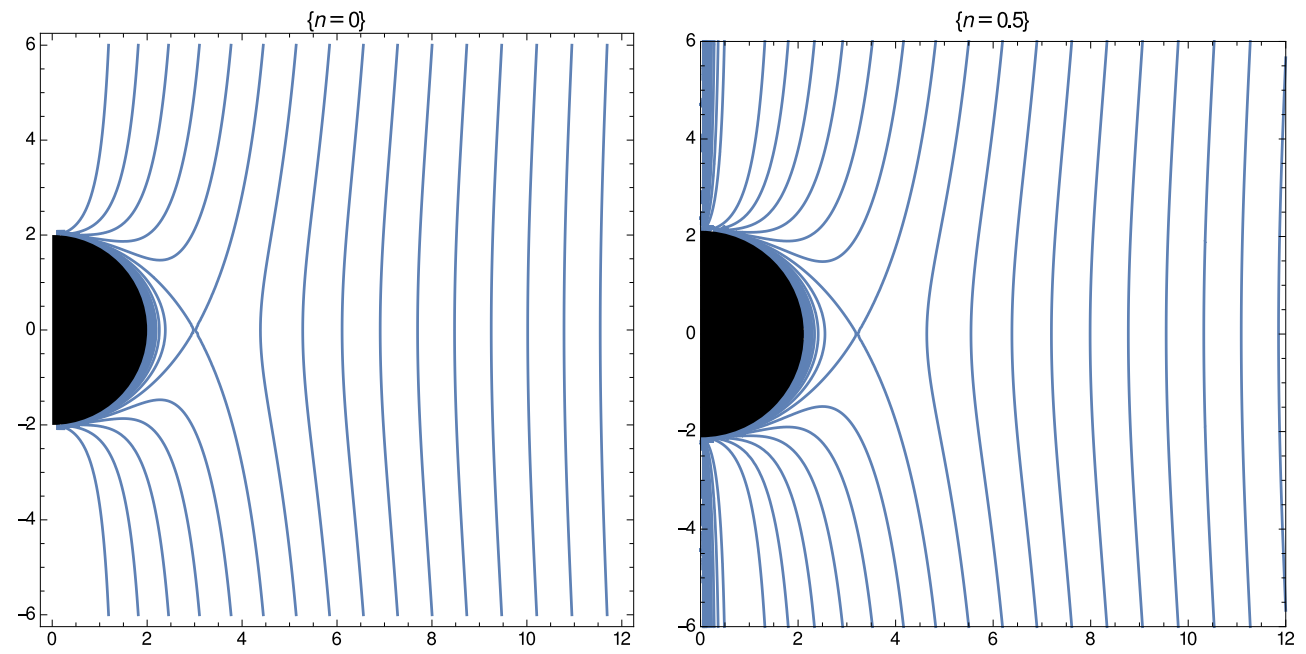

Figure 3. Von Zeipel cylinders with respect to the ZAMOs, $\tilde{\mathcal{R}}=$ constant, in the NUT space-time with $n=0.5 M$ and $C=0$ (right) and in the Schwarzschild spacetime (left). In these pictures and in all the following ones, $M$ is used as the unit on the axes.
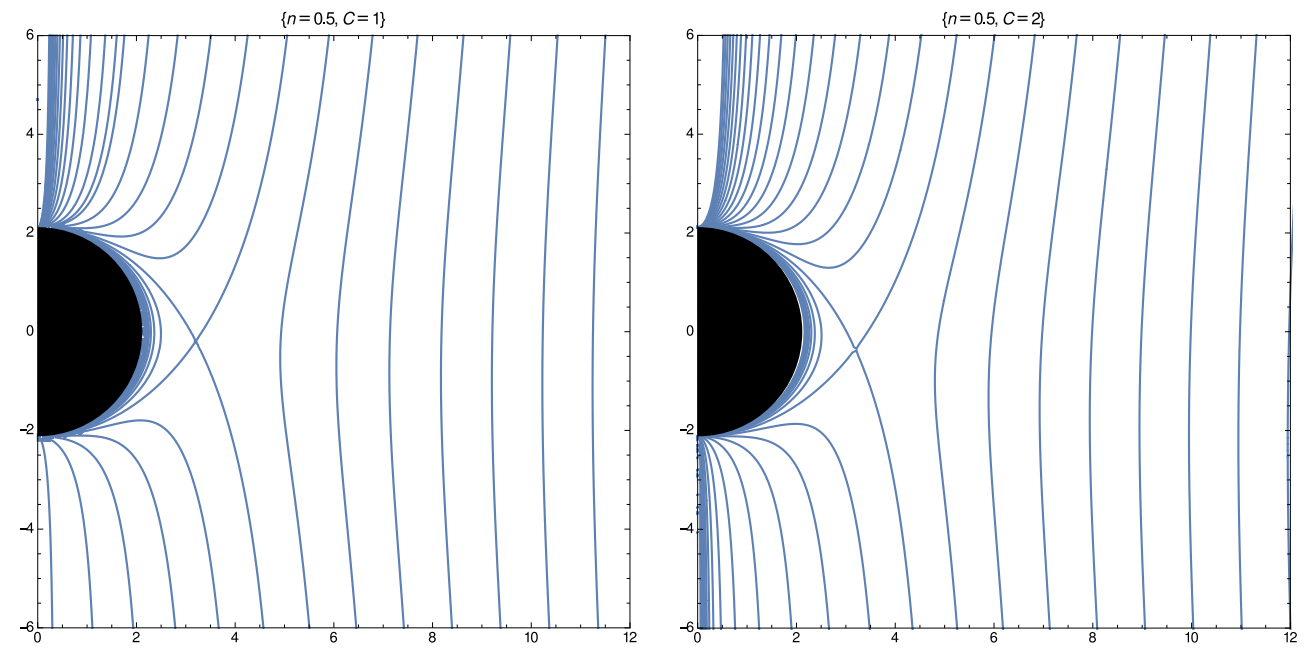

Figure 4. Von Zeipel cylinders with respect to the ZAMOs, $\tilde{\mathcal{R}}=$ constant, in the NUT space-time with $n=0.5 M$ and $C=1$ (left) and $C=2$ (right).

We now turn to the surfaces $\mathcal{R}=$ constant. In Figs. 5 we plot these surfaces in the NUT space-time and we compare them with those in the Kerr space-time which are shown in Fig. 6. As the surfaces $\mathcal{R}=$ constant are not universal, we have to specify the motion. This can be done by prescribing $\Omega$ or $\ell$ as a function of $r$ and $\theta$. We have chosen a motion with $\ell=$ constant which is the case we will also consider in Section 6 below, so the surfaces $\mathcal{R}=$ constant coincide with the surfaces $\Omega=$ constant. The Manko-Ruiz parameter is set equal to zero which is no restriction because we can reduce to this case by a transformation (40). We see that in the NUT space-time the von Zeipel cylinders with respect to the stationary observers are not symmetric with respect to the equatorial 

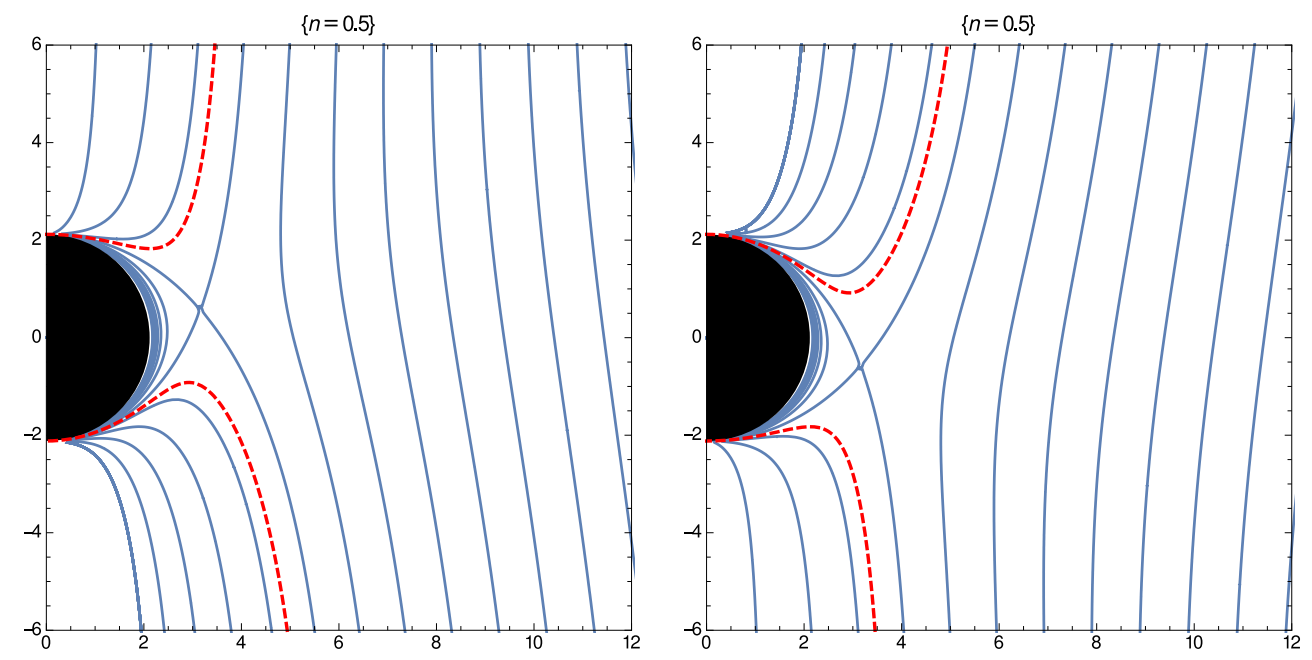

Figure 5. Von Zeipel cylinders with respect to the stationary observers, $\mathcal{R}=$ constant, in the NUT space-time with $n=0.5 M$ and $C=0$, for circular motion with $\ell=5 M$ (left) and $\ell=-5 M$ (right). The (red) dashed lines denote the surfaces where the fluid velocity becomes light-like; this is the boundary of the region where circular time-like motion with the chosen $\ell$ is possible. Reversing the sign of $\ell$ has the same effect as reversing the $z$-axis.

plane. Switching from $\ell$ to $-\ell$ has the same effect as changing $\theta$ into $\pi-\theta$. We have indicated by a (red) dashed line the boundary of the region where motion with the chosen $\ell$ is time-like, i.e., where the inequality (31) is satisfied. Formally, the function $\mathcal{R}$ is still well-defined outside of this region, because the right-hand side of (41) is still positive, but it is not associated with a physically reasonable motion; the right-hand side of (41) becomes negative only on a region near the half-axis $\theta=\pi$ for positive $\ell / n$ and near the half-axis $\theta=0$ for negative $\ell / n$. The situation is completely different in the Kerr metric, see Fig. 6. Here the von Zeipel cylinders with respect to the stationary observers are symmetric with respect to the equatorial plane. Changing the sign of $\ell$ does not correspond to reversing the $\mathrm{z}$ axis; clearly, the reason is that in the Kerr case the relative sign of $a$ and $\ell$ plays a role. Again, motion with the chosen $\ell$ is time-like only on part of the domain of outer communication, which is indicated by the (red) dashed line. In the Kerr case, the funtion $\mathcal{R}$ is well-defined, i.e., the right-hand side of (41) is positive, except for the co-rotating case $(\ell a>0)$ on a certain region near the horizon.

For our purpose, the most important property of the von Zeipel cylinders is in the fact that, for motions with $\ell=$ constant, the surfaces $\mathcal{R}=$ constant coincide with the surfaces $\Omega=$ constant. In addition, the von Zeipel cylinders are also related to inertial forces, as we will briefly discuss in the following section. 

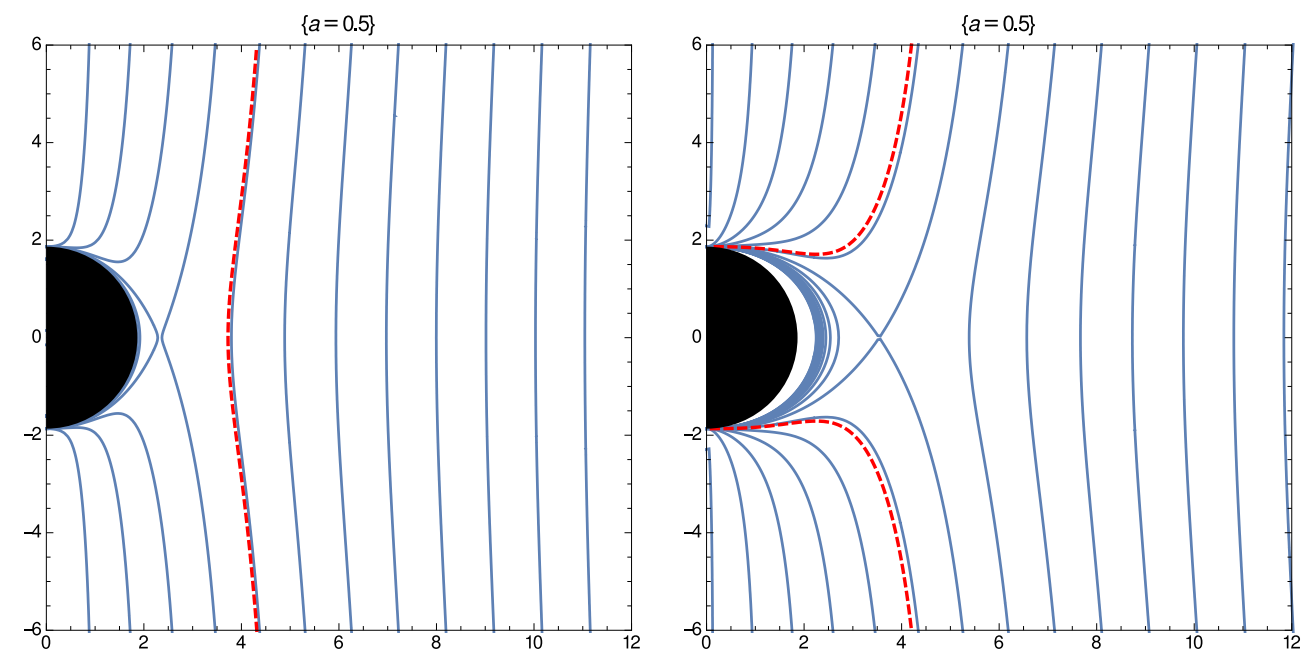

Figure 6. Von Zeipel cylinders with respect to the stationary observers, $\mathcal{R}=$ constant, in the Kerr space-time with $a=0.5 M$, for circular motion with $\ell=5 M$ (left) and $\ell=-5 M$ (right). The (red) dashed lines have the same meaning as in Fig. 6. As always, $M$ is used as the unit on the axes.

\section{Inertial forces}

In this section we want to discuss, for the kind of circular motion considered in the preceding section, the decomposition of the four-acceleration in terms of inertial accelerations (or inertial forces if we multiply with the mass of each particle). In particular, we want to discuss the relation of the von Zeipel cylinders to the inertial forces. As in the preceding section, we begin with some general results which hold for any axisymmetric and stationary metric before specifying the results for the NUT metric.

There are various ways in which the decomposition of the four-acceleration can be introduced. In any case, this decomposition depends on the choice of a kind of reference frame. This can be done on an arbitrary space-time, see Foertsch et al. [20], but here we are interested only in axisymmetric and stationary space-times of the form (29) where it is most convenient to introduce the tetrad

$$
e_{0}=\frac{\partial_{t}+\omega \partial_{\phi}}{\sqrt{g_{t t}+\omega g_{t \phi}}}, \quad e_{1}=\frac{1}{\sqrt{-g_{\phi \phi}}} \partial_{\phi}, \quad e_{2}=\frac{1}{\sqrt{-g_{\theta \theta}}} \partial_{\theta}, \quad e_{3}=\frac{1}{\sqrt{-g_{r r}}} \partial_{r},
$$

with $\omega$ being defined in (42). The integral curves of $e_{0}$ are the worldlines of the ZAMOs already considered in the preceding section; the tetrad (47) is also known as the locally non-rotating frame (LNRF). We will decompose, following Abramowicz et al. [18], the four-acceleration of particles in circular motion with respect to this tetrad.

We begin by rewriting the four-velocity (30) of our particles as

$$
u=\frac{e_{0}+v e_{1}}{\sqrt{1-v^{2}}},
$$

where $v$ is a function of $r$ and $\theta$ that gives, at each point, the three-velocity as measured 
by a ZAMO. We distinguish between the four-acceleration as a vector, $a$, and the fouracceleration as a covector, $A$, which are related by

$$
A=g(a, \cdot), \quad a=\nabla_{u} u .
$$

The inertial acceleration is the acceleration of freely falling particles relative to our particles, i.e., it is given by the covector $-A$. From (48) and (49) we find that the inertial acceleration can be decomposed in the form

$$
-A=A_{\mathrm{gr}}+A_{\mathrm{Cor}}+A_{\mathrm{cen}}
$$

where the first term is independent of $v$, the second term is odd in $v$ and the third term is even in $v$,

$$
\begin{aligned}
& A_{\text {gr }}=-\frac{1}{2} \mathrm{~d} \ln \left(-g_{t t}-\omega\right), \\
& A_{\text {Cor }}=-\frac{v}{1-v^{2}} \tilde{\mathcal{R}} \mathrm{d} \omega, \\
& A_{\text {cen }}=\frac{v^{2}}{\left(1-v^{2}\right)} \tilde{\mathcal{R}}^{-1} \mathrm{~d} \tilde{\mathcal{R}}
\end{aligned}
$$

cf. Abramowicz et al. [18]. $A_{\text {gr }}, A_{\text {Cor }}$ and $A_{\text {cen }}$ are called the gravitational acceleration, the Coriolis acceleration and the centrifugal acceleration, respectively. Note that each of the three expressions (51), (52) and (53) is proportional to a differential, i.e., that $a_{\mathrm{gr}}, a_{\mathrm{Cor}}$ and $a_{\text {cen }}$ are each perpendicular to a family of potential surfaces.

Recall that in general $\mathcal{R}$ depends on $v$ whereas $\tilde{\mathcal{R}}$ does not. We will now investigate how $\mathcal{R}$ behaves in the ultrarelativistic limit, i. e. for $v \rightarrow \pm 1$. If we epxress $\Omega$ in terms of $v$,

$$
\Omega=\omega-\frac{\sqrt{g_{t \phi}^{2}-g_{t t} g_{\phi \phi}}}{g_{\phi \phi}} v=\omega+\frac{v}{\tilde{\mathcal{R}}},
$$

we find after a straight-forward calculation that the two definitions of von Zeipel radii (37) and (41) are related by

$$
\mathcal{R}_{ \pm}:=\lim _{v \rightarrow \pm 1} \mathcal{R}=\frac{\tilde{\mathcal{R}}}{1 \pm \omega \tilde{\mathcal{R}}}
$$

If we compare the expression

$$
d \mathcal{R}_{ \pm}=\frac{d \tilde{\mathcal{R}} \mp \tilde{\mathcal{R}}^{2} d \omega}{(1 \pm \omega \tilde{\mathcal{R}})^{2}}
$$

with the fact that

$$
A_{\text {Cor }}+A_{\text {cen }}=\frac{v^{2} Z(v)}{\left(1-v^{2}\right) \tilde{\mathcal{R}}}, \quad Z(v)=d \tilde{\mathcal{R}}-\frac{1}{v} \tilde{\mathcal{R}}^{2} d \omega,
$$

we see that in the ultrarelativistic limit the sum of Coriolis and centrifugal acceleration becomes perpendicular to the potential surfaces $\mathcal{R}_{ \pm}=$constant, cf. Hasse and Perlick [21] where this result was already found for the special case of the Kerr-Newman space-time. If $g_{t \phi}=0$ we have of course $\mathcal{R}_{ \pm}=\tilde{\mathcal{R}}=\mathcal{R}$ and $a_{\text {Cor }}+a_{\text {cen }}=a_{\text {cen }}$ is perpendicular to the surfaces $\mathcal{R}=$ constant for any $v$. 

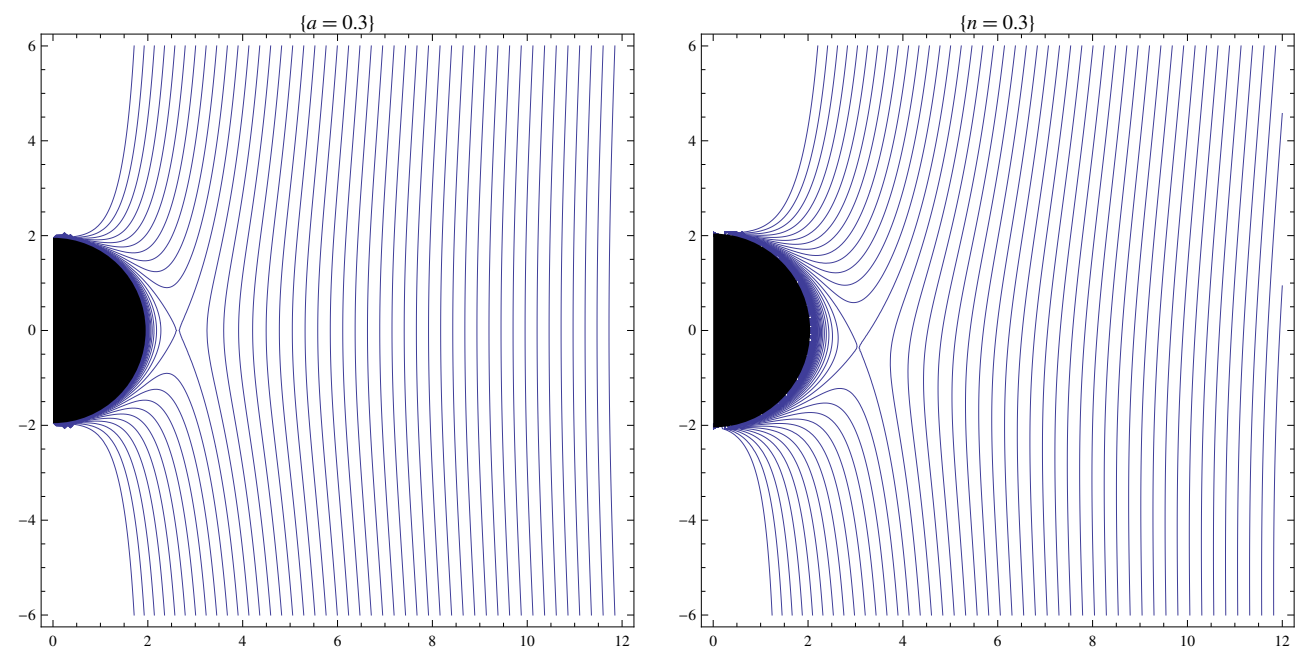

Figure 7. The potential surfaces for the ultrarelativistic limit of centrifugal-plusCoriolis force $\mathcal{R}_{+}=$constant in Kerr space-time with $a=0.3 M$ (left) and in NUT space-time with $n=0.3 M$ (right).

In the NUT space-time the potential $\mathcal{R}_{ \pm}$is given by

$$
\mathcal{R}_{ \pm}=\frac{\left(r^{2}+n^{2}\right) \sin \theta}{\sqrt{r^{2}-2 M r-n^{2}}} \pm 2 n(\cos \theta+C) .
$$

As $C$ enters only in terms of an additive constant, the set of surfaces $\mathcal{R}_{ \pm}=$constant is independent of $C$. For the co-rotating case, i.e., for the plus sign, these surfaces are plotted in Fig. 7, in comparison with the Kerr metric. In the NUT and in the Kerr case, there is exactly one value $(r, \theta)$ where $d \mathcal{R}_{ \pm}=0$. This indicates a circular light-like geodesic, cf. Fig. 2.

\section{Polish doughnuts with constant specific angular momentum in NUT space-time}

The so-called Polish doughnuts are analytical solutions for stationary fluid flows orbiting a black hole. They have been introduced by Jaroszyński, Abramowicz and Paczyński [22] on Schwarzschild and on Kerr space-time; a detailed discussion can be found, e.g., in the book by Rezzolla and Zanotti [17]. Here we want to discuss Polish doughnuts on the NUT space-time to see how their shape is influenced by the NUT parameter.

We consider circular fluid flows, i.e., fluid flows with a four-velocity of the form (30) with $\Omega$ being a function of $r$ and $\theta$. We asume that it is a perfect fluid with energy-momentum tensor

$$
T^{\mu \nu}=(e+p) u^{\mu} u^{\nu}-p g^{\mu \nu},
$$

where $e$ is the energy density and $p$ is the pressure. This fluid will obey the Euler equation which for circular motion can be written as

$$
\frac{\partial_{\mu} p}{\rho h}=-\partial_{\mu} \ln \left(u_{t}\right)+\frac{\Omega \partial_{\mu} \ell}{1-\Omega \ell}=a_{\mu}
$$


with $\ell$ being the specific angular momentum defined in (32), $\rho$ being the mass density and $h:=(e+p) / \rho=1+\epsilon+p / \rho$ being the specific enthalpy. $\epsilon$ is the density of the internal energy. We will consider the case that the fluid satisfies a barotropic equation of state. We have already mentioned above the relativistic von Zeipel theorem which says that then the differential $d \Omega$ is proportional to the differential $d \ell$ provided that the latter is non-zero. Below, however, we will consider the case that $\ell$ is a constant, i.e., that $d \ell$ is everywhere equal to zero. In this case, the assumption of the von Zeipel theorem does not hold; however, the surfaces $\Omega=$ constant coincide with the von Zeipel cylinders $\mathcal{R}=$ constant.

The idea of constructing Polish doughnut solutions is based on the observation that (60) can be integrated, assuming a barotropic equation of state and making use of the fact that the pressure vanishes on the surface of the fluid body. This results in

$$
W-W_{\text {in }}:=-\int_{0}^{p} \frac{d p^{\prime}}{\rho h}=\ln \left(u_{t}\right)-\ln \left(\left(u_{t}\right)_{\text {in }}\right)-\int_{\ell_{\text {in }}}^{\ell} \frac{\Omega d \ell^{\prime}}{1-\Omega \ell^{\prime}},
$$

where $W_{\text {in }}$ is a constant of integration. In general, $\ell$ is a function of $r$ and $\theta$. However, the simplest idea is to look for solutions where $\ell=$ const. In this particular case the integral on the right-hand side of (61) vanishes and imposing the condition that $W \rightarrow 0$ for $r \rightarrow \infty$ we obtain in the NUT space-time

$$
W=\ln \left(u_{t}\right)=\frac{1}{2} \ln \left(\frac{\Delta \Sigma \sin ^{2} \theta}{\Sigma^{2} \sin ^{2} \theta-\Delta(\ell-2 n C-2 n \cos \theta)^{2}}\right) .
$$

Here we have used the normalisation condition of the four-velocity to express $u_{t}$ as

$$
u_{t}=\sqrt{\frac{g_{t \phi}^{2}-g_{t t} g_{\phi \phi}}{-g_{\phi \phi}-2 g_{t \phi} \ell-g_{t t} \ell^{2}}} .
$$

For each value of $\ell$, we have to restrict to the space-time region where the condition (34) for time-like motion is satisfied. On this region, the von Zeipel cylinders $\mathcal{R}=$ constant give the surfaces of constant $\Omega$.

Specifying further to a polytropic equation of state

$$
p=K \rho^{\Gamma}
$$

and, correspondingly,

$$
\epsilon=\frac{K \rho^{\Gamma-1}}{\Gamma-1}
$$

with $K$ and $\Gamma$ being constants, we can compute the pressure integral in (61) and obtain for the density distribution of the matter:

$$
\rho(r, \theta)=\left(\frac{\Gamma-1}{K \Gamma}\left(\exp \left(W_{\text {in }}-W(r, \theta ; \ell)\right)-1\right)\right)^{1 /(\Gamma-1)} .
$$

The perfect fluid fills the region where $\rho \geq 0$, i.e., where $W(r, \theta ; \ell) \leq W_{\text {in. }}$. The construction of Polish doughnut solutions with $\ell=$ constant depends on two free parameters, the specific angular momentum $\ell$ and the integration constant $W_{\text {in }}$. Different values one may choose for the constant $\ell$ define different geometrical 
configurations of the rotating matter (see e. g. [23]). The constant $W_{\text {in }}$ determines which of the equipotential surfaces is the boundary of the fluid. Note that the shape of the Polish doughnut is independent of $\Gamma$ and $K$.

The only place where the Manko-Ruiz parameter $C$ enters into this construction is in the potential (62). We see that changing from $C$ to $C^{\prime}$ can be compensated for by changing the constant $\ell$ to $\ell^{\prime}=\ell+2 n\left(C^{\prime}-C\right)$. This demonstrates that it is no restriction of generality if we assume $C=0$ for the rest of this section.

From (60) and (62) we read that our assumption $\partial_{\mu} \ell=0$ implies that $a_{\mu}=-\partial_{\mu} W$, so the motion is geodesic at the critical points of $W$. We could determine these points by differentiating (62). As an alternative, we may use the equation $\ell=L / E$ and insert the expressions (18) and (19) for geodesic motion. As we assume $C=0$, this results in

$$
\ell=\frac{2 n}{\cos \theta} .
$$

With $\cos ^{2} \theta$ given by (21), we get the "Keplerian" (i.e., geodesic) specific angular momentum as a function of $r$,

$$
\ell_{K}(r)^{2}=Q(r), \quad r_{\mathrm{ph}}<r<\infty,
$$

where $Q(r)$ is defined in (22). Depending on the value of $\ell$ the equation $\ell=\ell_{K}(r)$ can have none, one or two solutions $r$ outside of the horizon. Astrophysically interesting configurations occur when there are two such solutions, a local minimum and a saddle of $W$. This is the case if $\ell_{K}\left(r_{\mathrm{ms}}\right)<\ell<\ell_{K}\left(r_{\mathrm{mb}}\right)$. (Recall that $r_{\mathrm{ms}}$ and $r_{\mathrm{mb}}$ are determined by (26) and (28), respectively.) The first inequality $\ell_{K}\left(r_{\mathrm{ms}}\right)<\ell$ guarantees that $W$ has closed equipotential surfaces and a local minimum at a centre with radius coordinate $r_{\text {cen }}$. There the density and, thus, the pressure reaches a maximum and the matter moves on a geodesic, see Fig. 8 for a plot of the acceleration near $r_{\text {cen }}$. If $\ell_{K}\left(r_{\mathrm{ms}}\right)<\ell<\ell_{K}\left(r_{\mathrm{mb}}\right)$, the region with closed equipotential surfaces is bounded by an equipotential surface that forms a "cusp" at a radius coordinate $r_{\mathrm{c}}$ which lies between $r_{\text {hor }}$ and $r_{\text {cen }}$. The $\theta$ coordinates of the centre and of the cusp are to be determined by inserting $r_{\text {cen }}$ and $r_{\mathrm{c}}$, respectively, into $(21)$.

The choice of a value for $W_{\text {in }}$ defines the size of the configuration and whether or not it has an inner edge, see [23] and [17] for more details. In the case $\ell_{K}\left(r_{\mathrm{ms}}\right)<\ell<\ell_{K}\left(r_{\mathrm{mb}}\right)$, where we have a centre and a cusp, an inner edge exists if and only if the cusp is outside the fluid configuration, i.e., if and only if $W\left(r_{\text {cen }}, \theta_{\text {cen }}\right)<W_{\text {in }}<W\left(r_{\mathrm{c}}, \theta_{\mathrm{c}}\right)$. Then the boundary of the fluid is topologically a torus, as the name Polish "doughnut" suggests. The inner and the outer edge of the torus are determined by the equations

$$
W=W_{\text {in }}, \quad \partial_{\theta} W=0 .
$$

Differentiating (62) with respect to $\theta$, assuming $C=0$, demonstrates that the second condition is equivalent to (67), i.e., the edges are on the surface of time-like circular geodesic orbits (although they are non-geodesic). In particular, the coordinates $r_{\text {in }}$ and $\theta_{\text {in }}$ of the inner edge are related by (21). It is then equivalent for the toroidal configuration to use instead of the parameters $\ell$ and $W_{\text {in }}$ the two radii $r_{\text {cen }}$ and $r_{\text {in }}$. If 
these two radii are given, the values of $\ell$ and $W_{\text {in }}$ to be inserted into (66) are $\ell=\ell_{K}\left(r_{\text {cen }}\right)$ and $W_{\text {in }}=W\left(r_{\text {in }}, \theta_{\text {in }} ; \ell_{K}\left(r_{\text {cen }}\right)\right)$.

The most important new feature of a Polish doughnut in the NUT space-time, in comparison to the Schwarzschild or Kerr case, is in the fact that the symmetry with respect to the equatorial plane is broken. In particular the centre, the cusp and the edges are not in the equatorial plane but rather in the surface of circular time-like geodesic orbits.

As we have solved the Euler equation on a fixed space-time background, the model is consistent only if the back-reaction of the fluid onto the space-time geometry is negligible. We check that this is true by calculating the total mass of the torus and comparing it with the mass of the black hole, and also by calculating the density and comparing it with a typical curvature invariant.

The mass $m$ of the torus is computed using the standard generally relativistic expression

$$
m=\int_{V} T_{0}^{0} \sqrt{-g} \mathrm{~d} V=2 \pi \int \rho(r, \theta) \sqrt{-g} \mathrm{~d} r \mathrm{~d} \theta .
$$

We have to choose the parameters such that $m$ is small in comparison to $M$.

As a typical curvature invariant we use the Kretschmann scalar which is given in the NUT space-time by

$$
\begin{aligned}
K_{S} & =R_{\alpha \beta \gamma \delta} R^{\alpha \beta \gamma \delta} \\
& =\frac{48\left(\left(M^{2}-n^{2}\right)\left(r^{6}-15 n^{2} r^{4}+15 n^{4} r^{2}-n^{6}\right)+4 M n^{2} r\left(3 r^{4}-10 n^{2} r^{2}+3 n^{4}\right)\right)}{\left(r^{2}+n^{2}\right)^{6}}
\end{aligned}
$$

where $R_{\beta \gamma \delta}^{\alpha}$ is the Riemannian curvature tensor. It can be checked that the Kretschmann scalar is strictly positive outside the horizon. As a test that the influence of the fluid on the background geometry is negligible, we compare at the centre of the torus the square of the density with the Kretschmann scalar. Demanding that $\left.\left.\rho^{2}\right|_{r_{\text {cen }}} \ll K_{S}\right|_{r_{\text {cen }}}$ poses restrictions on the remaining free parameters $K$ and $\Gamma$ of the equation of state. Choosing the gas to be non-relativistic we fix the value of $\Gamma=5 / 3$. Then the desired inequality restricts the possible values of $K$ by

$$
K \gg \frac{\Gamma-1}{\Gamma K_{S}^{(\Gamma-1) / 2}}\left(\exp \left(W_{\text {in }}-W\left(r_{\text {cen }}, \theta_{\text {cen }} ; \ell\right)\right)-1\right) .
$$

Assuming the equation of state for a non-relativistic ideal gas, $p=\frac{\rho}{\mu} T$, with $\mu$ being the mass of a molecule in the gas, one can also find the temperature distribution inside the torus,

$$
T=K \rho^{\Gamma-1} \mu \text {. }
$$

We have calculated the temperature at the centre of the torus for our numerical examples, see Table 1, where we assumed a hydrogen gas, i.e., we inserted for $\mu$ the proton mass. We see that the temperature is fairly high, but the so-called coolness parameter $\mu c^{2} /(k T)$ is still large enough so that the assumption of a non-relativistic ideal gas may be viewed as acceptable. Also note that, for our torus which was constructed 


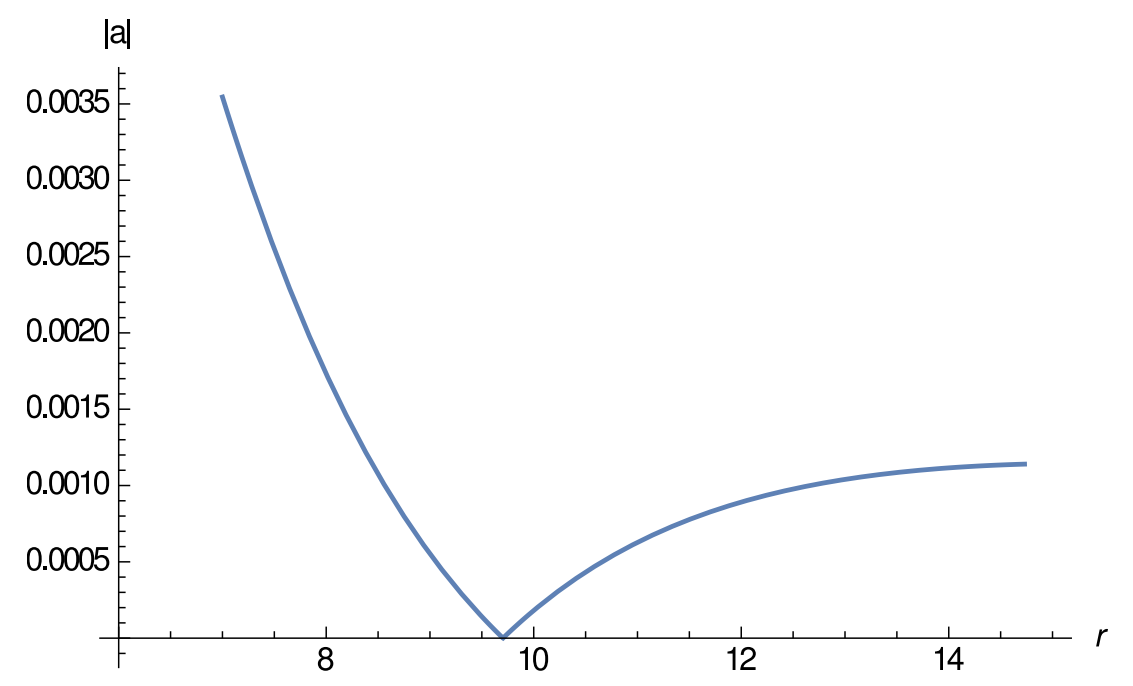

Figure 8. Modulus $|a|=\sqrt{a_{\mu} a^{\mu}}$ of the acceleration $a_{\mu}$ from (60) as a function of $r$ on the surface of circular geodesic orbits inside a torus with parameters $r_{\text {cen }}=9.7 M$, $r_{\text {in }}=7 M$. The radius coordinate $r$ is given in units of $M$ and the acceleration $|a|$ is given in units of $M^{-1}$.

with $r_{\text {cen }}$ and $r_{\text {in }}$ given in units of the black-hole mass $M$, the temperature at the centre is independent of $M$.

In Fig. 9 we show Polish doughnuts in NUT space-time for two different values of the NUT parameter and, for the sake of comparison, also in Schwarzschild and Kerr space-times. In all four cases, we have chosen the same values for $r_{\text {in }}$ and $r_{\text {cen }}$. The total mass of the torus and the density at the centre are given in Table 1. The distinguishing feature of a Polish doughnut in NUT space-time is the asymmetry with respect to the equatorial plane. The mathematical construction gives a positive density inside the torus and also in a second region adjacent to the horizon. For the sake of completeness, we have included this second region in Fig. 9. However, we do not believe that is has any physical relevance because matter on a circular orbit so close to the horizon is expected to fall into the black hole under the slightest perturbation. Also, with the chosen parameters the mass of the fluid inside this region is so big that it could not be neglected in comparison to the mass of the black hole. For these reasons, we assume that this second fluid configuration is actually not present and we did not include it into the calculation of the configuration mass (70).

\section{Conclusions}

In this article we have investigated circular motion of particles in the domain of outer communication of a NUT black hole. A major difference in comparison to the case of a Schwarzschild or Kerr black hole is in the fact that the geometry is no longer symmetric with respect to the equatorial plane. This asymmetry is reflected by the fact that circular time-like geodesics are not in the equatorial plane but rather in a curved 

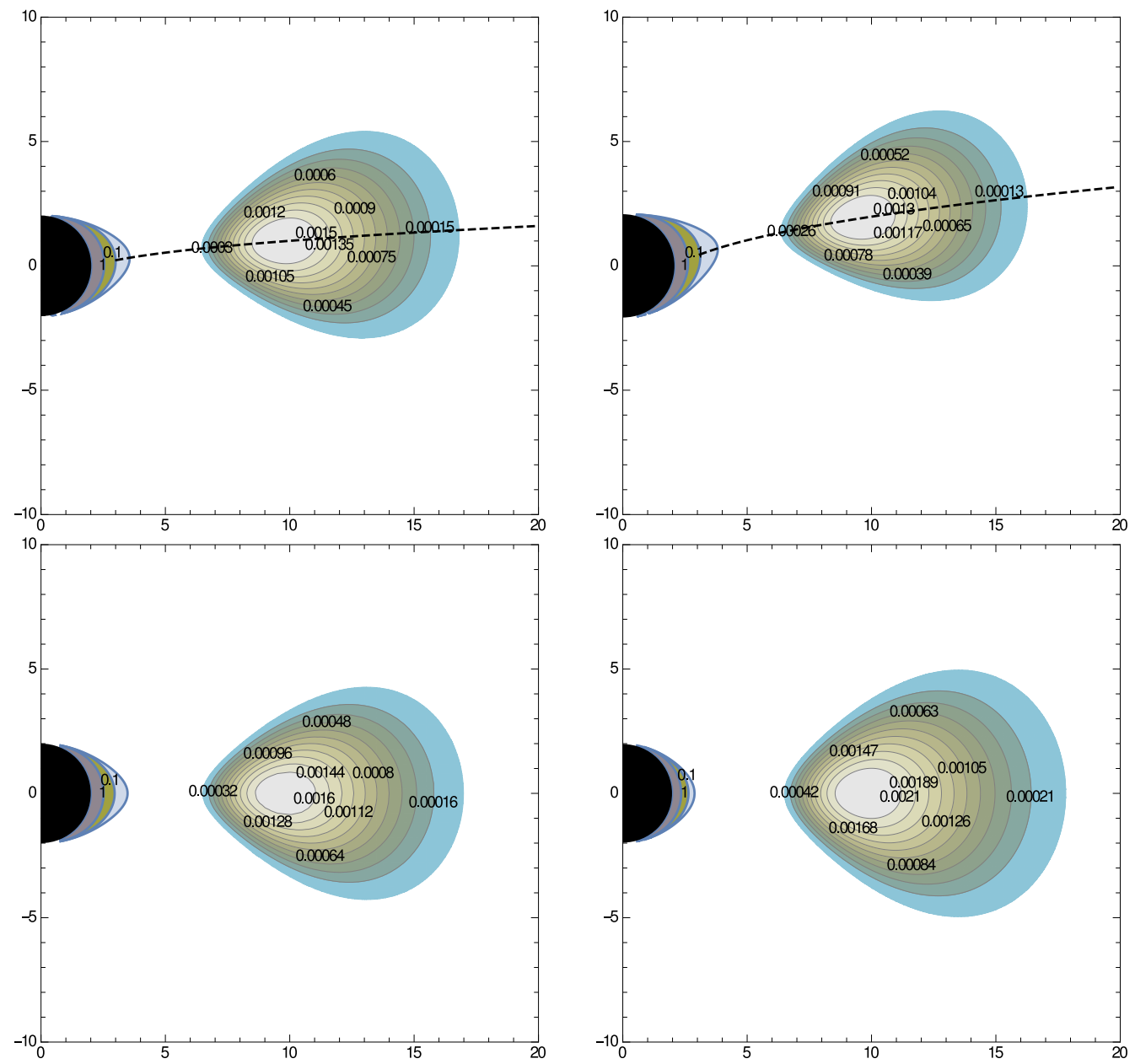

Figure 9. Polish Doughnut with $r_{\text {cen }}=9.7 M$ and $r_{\text {in }}=6.5 M$ in NUT spacetime with $n=0.2 M$ (top left), in NUT space-time with $n=0.4 M$ (top right), in Schwarzschild space-time (bottom left) and in Kerr space-time with $a=0.2 M$ (bottom right). As always, we use $M$ as the unit on the axes. Different colours correspond to different values of the matter density $\rho$ given in units of $1 / M^{2}$. A polytropic equation of state (64) is assumed with $\Gamma=5 / 3$ and $K=0.2$ which satisfies the inequality (72) for all the cases considered. In the top figures the dashed line marks the surface of circular geodesic orbits. In addition to the torus there is a second region, near the horizon, where the mathematical construction gives a positive density. We have included it in the picture but, as explained in the text, it is to be considered as unphysical.

\begin{tabular}{|c|c|c|c|c|}
\hline$n / M$ & $a / M$ & $m /\left(10^{-2} M\right)$ & $\rho_{\text {cen }} /\left(10^{-4} M^{-2}\right)$ & $T_{\text {cen }} /\left(10^{9} K\right)$ \\
\hline 0 & 0 & 5.71 & 2.05 & 7.46 \\
\hline 0.2 & 0 & 5.28 & 1.99 & 7.01 \\
\hline 0.4 & 0 & 7.74 & 1.81 & 7.60 \\
\hline 0 & 0.2 & 7.91 & 2.52 & 8.74 \\
\hline
\end{tabular}

Table 1. Torus mass $m$, density in the centre $\rho_{\text {cen }}$ and temperature in the centre for different values of NUT and Kerr parameters. The values for $n, a, m, \rho_{c e n}$ are given in terms of the black-hole mass $M$, the temperature is given in Kelvins. 
surface. This could be observed, e.g. if a NUT source is surrounded by a thin disc of dust (a "Saturn ring"). We have shown that the same asymmetry can be seen in thick accretion tori known as Polish doughnuts. Our results on the surface of circular time-like orbits in three-dimensional space and on the shapes of Polish doughnuts apply to Misner's interpretation of the NUT space-time (with a periodic time coordinate) and equally well to Bonnor's interpretation (with a singularity on at least one half-axis). Also, these results are independent of the Manko-Ruiz parameter $C$.

For the sake of illustration we have used von Zeipel cylinders with respect to the stationary observers, $\mathcal{R}=$ constant, and with respect to the ZAMOs, $\tilde{\mathcal{R}}=$ constant. Of all the features discussed in this paper, the surfaces $\tilde{\mathcal{R}}=$ constant are the only ones that have an essential dependence on the Manko-Ruiz parameter $C$. The reason is that the ZAMOs depend on $C$ : A (local) coordinate transformation that transforms $C$ to $C^{\prime}$ changes the surfaces $t=$ constant and thus the ZAMOs.

As we have said in the introduction, the question of whether NUT sources exist in Nature is a matter of debate. However, if they do exist, they could be detected by way of their lensing features, as shown by Nouri-Zonoz and Lynden-Bell [9], and also by way of their influence on orbiting matter, as shown in this paper.

\section{Acknowledgements}

P. I. Je. was financed through the PhD-student programme Erasmus Mundus Joint Doctorate in International Relativistic Astrophysics (EMJD IRAP) during the course of this work. Moreover, we gratefully acknowledge support from the DFG within the Research Training Group 1620 "Models of Gravity".

\section{References}

[1] Newman E T, Tamburino L and Unti T W J 1963 J. Math. Phys 4 915-923

[2] Bradley M, Fodor G, Gergely L, Marklund M and Perjés Z 1999 Class. Quantum Grav. 16 16671675

[3] Taub A H 1951 Ann. Math. 53 472-490

[4] Misner C 1963 J. Math. Phys 4 924-937

[5] Bonnor W B 1969 Math. Proc. Cambr. Philos. Soc. 66 145-151

[6] Manko V S and Ruiz E 2005 Class. Quantum Grav. 22 3555-3560

[7] McGuire P and Ruffini R 1975 Phys. Rev. D 12 3026-3029

[8] Misner C 1967 in Ehlers J (ed) Relativity theory and astrophysics.I. (Amer. Math. Soc., Providence, Rhode Island) p. 160

[9] Nouri-Zonoz M and Lynden-Bell D 1997 Mon. Not. Roy. Soc 292 714-722

[10] Grenzebach A, Perlick V and Lämmerzahl C 2014 Phys. Rev. D 89124004

[11] Kagramanova V, Kunz J, Hackmann E and Lämmerzahl C 2010 Phys. Rev. D 81124044

[12] Hackmann E and Lämmerzahl C 2012 Phys. Rev. D 85044049

[13] Griffiths J B and Podolský J 2009 Exact Space-Times in Einstein's General Relativity (Cambridge UP)

[14] Chakraborty C 2014 Eur. Phys. J. C $\mathbf{7 4} 2759$

[15] Abramowicz M A 1971 Acta Astron. 21 81-85

[16] Abramowicz M A, Miller J C and Stuchlík Z 1993 Phys. Rev. D 47 1440-1447 
[17] Rezzolla L and Zanotti O 2013 Relativistic Hydrodynamics (Oxford University Press)

[18] Abramowicz M A, Nurowski P and Wex N 1995 Class. Quantum Grav. 12 1467-1472

[19] Stuchlík Z, Slaný P, Török G and Abramowicz M A 2005 Phys. Rev. D 71024037

[20] Foertsch T, Hasse W and Perlick V 2003 Class. Quantum Grav. 20 4635-4651

[21] Hasse W and Perlick V 2006 J. Math. Phys. 47042503

[22] Jaroszyński M, Abramowicz M A and Paczyński B 1980 Acta Astron. 30 1-34

[23] Font J A and Daigne F 2002 Mon. Not. R. Astron. Soc. 334 383-400 